\title{
A high-throughput drug combination screen identifies an anti-glioma synergism between TH588 and PI3K inhibitors
}

\author{
Zhen Chen ${ }^{1}$, Chao Chen ${ }^{1}$, Tingting Zhou ${ }^{1}$, Chao Duan' ${ }^{1}$ Qianqian Wang ${ }^{2}$, Xiaohui Zhou' ${ }^{1}$ Xia Zhang ${ }^{1}$, \\ Fangrong $\mathrm{Wu}^{1}$, Yunfen $\mathrm{Hua}^{3}$ and Fan $\mathrm{Lin}^{1,4^{*}}$ (D)
}

\begin{abstract}
Background: Glioblastoma multiforme (GBM) is the most common and lethal type of primary brain tumor. More than half of GBMs contain mutation(s) of PTEN/PI3K/AKT, making inhibitors targeting the PI3K pathway very attractive for clinical investigation. However, so far, PI3K/AKT/mTOR inhibitors have not achieved satisfactory therapeutic effects in clinical trials of GBM. In this study, we aimed to develop a high-throughput screening method for high-throughput identification of potential targeted agents that synergize with PI3K inhibitors in GBM.

Methods: A Sensitivity Index (SI)-based drug combination screening method was established to evaluate the interactions between BKM120, a pan-PI3K inhibitor, and compounds from a library of 606 target-selective inhibitors. Proliferation, colony and 3D spheroid formation assays, western blotting, comet assay, $\mathrm{Y}-\mathrm{H} 2 \mathrm{AX}$ staining were used to evaluate the anti-glioma effects of the top-ranked candidates. The drug combination effects were analyzed by the Chou-Talalay method.
\end{abstract}

Results: Six compounds were successfully identified from the drug screen, including three previously reported compounds that cause synergistic antitumor effects with PI3K/mTOR inhibitors. TH588, an putative MTH1 inhibitor exhibited significant synergy with BKM120 in suppressing the proliferation, colony formation and 3D spheroid formation of GBM cells. Further investigation revealed that both DNA damage and apoptosis were markedly enhanced upon combination treatment with TH588 and BKM120. Finally, activation of PI3K or overexpression of AKT compromised the anti-glioma efficacy of TH588.

Conclusions: The screening method developed in this study demonstrated its usefulness in the rapid identification of synergistic drug combinations of PI3K inhibitors and targeted agents.

Keywords: Glioblastoma, Sensitivity index, PI3K, MTH1, TH588

\section{Background}

Glioblastoma multiforme (GBM) is the most common and lethal type of brain cancer in adults. Current first-line therapy for GBM is a combination of surgery,

\footnotetext{
${ }^{*}$ Correspondence: linfee@me.com

${ }^{1}$ Department of Cell Biology, School of Basic Medical Sciences, Nanjing

Medical University, XueHai Building A111, 101 Longmian Avenue, Nanjing, Jiangning District, China

Full list of author information is available at the end of the article
}

chemotherapy and radiotherapy [1]. Despite such aggressive treatment, the overall survival of treated GBM patients is still shorter than 15 months [2], so the development of novel and effective therapy for GBM is a dire need. However, in the last decade, most clinical trials evaluating new therapeutics in GBM have encountered failures. Major issues need to be solved in the development of future anti-GBM therapy including but not limited to: the insufficient delivery of drug to the brain,

(c) The Author(s) 2020. This article is licensed under a Creative Commons Attribution 4.0 International License, which permits use, sharing, adaptation, distribution and reproduction in any medium or format, as long as you give appropriate credit to the original author(s) and the source, provide a link to the Creative Commons licence, and indicate if changes were made. The images or other third party material in this article are included in the article's Creative Commons licence, unless indicated otherwise in a credit line to the material. If material is not included in the article's Creative Commons licence and your intended use is not permitted by statutory regulation or exceeds the permitted use, you will need to obtain permission directly from the copyright holder. To view a copy of this licence, visit http://creativeco mmons.org/licenses/by/4.0/. The Creative Commons Public Domain Dedication waiver (http://creativecommons.org/publicdomain/ zero/1.0/) applies to the data made available in this article, unless otherwise stated in a credit line to the data. 
genetically heterogeneous nature of GBM, and aberrant activation of multiple signal pathways [3].

The phosphatidylinositol 3-kinase (PI3K) pathway affects several cellular processes that are essential for cancer progression, including cell growth, survival, motility, DNA repair and metabolism [4]. In GBM, several components of the PI3K pathway are frequently mutated. According to the TCGA GBM project, the frequency of genetic alterations in the PI3K pathway, such as mutation or amplification of EGFR, activated mutations of PI3CA (p110) or PIK3R1 (P85), and loss of PTEN, were estimated to occur in up to $88 \%$ of GBMs [5]. Therefore, targeting PI3K and its downstream effectors, such as AKT and mTOR, seems to be a promising strategy for treating GBM. Indeed, a variety of targeted agents in PI3K pathways are currently under evaluation in different stages of clinical trials [6].

Unfortunately, most agents targeting the PI3K-AKTmTOR pathway have failed to achieve satisfactory effects in the treatment of GBM so far [7, 8]. Modest monotherapy activity, unexpected statistically significant toxicity, and limited benefits in survival have been observed for PI3K inhibitors [9-11]. Thus, deep insights into the bottleneck of the anti-glioma effect of PI3K inhibitor treatment are needed. For example, recent research showed that drug resistance is likely driven by compensatory activation of alternative pathways and reorganization of protein networks [12]. Moreover, given the importance of the PI3K pathway in GBM genesis and development as well as the high incidence of mutations in this pathway, novel strategies for more effective combination regimens should be explored to enhance the anti-GBM effect of PI3K inhibitors. Importantly, synergistic combination therapies can improve efficacy, reduce the dose of individual drugs, and avoid potential drug toxicity [13].

In this study, we aimed to develop a method for efficiently identifying targeted agents that work with PI3K inhibitors in synergy to suppress the growth of GBM cells in a high-throughput format. We selected BKM120, a pan-PI3K inhibitor of all catalytic subunit isoforms of class I PI3Ks (p110 $\alpha, \mathrm{p} 110 \beta$, and p110 $\gamma)$. Interestingly, BKM120 can cross the blood-brain barrier relatively easy because its brain penetration is not restricted by $A B C B 1$ and $A B C G 2$, the two drug-efflux transporters responsible for elimination of most exogenous compounds in the brain. Moreover, invasion to the surrounding tissue is an important feature of GBM and that could be effectively inhibited by BKM120. Therefore, BKM120 represents an attractive candidate drug for GBM treatment [14-17].

To find the agents may sensitize the anti-glioma effect of BKM120 with known targets, we chose a drug library composing of target selective small molecular inhibitors. These small molecules targeting signaling proteins involved in several well-described signaling pathways, including neuronal signaling, metabolism, epigenetics, DNA damage, etc.

Lastly, it would be a laborious work if we want to identify drug combinations with synergistic effects from hundreds of compounds using the conventional doseresponse matrix method. Instead, we developed a novel strategy that only the effect of one a fixed concentration of BKM120 need to be tested. This strategy allows highthroughput screening and quick discovery of potential useful combination therapies for GBM treatment.

\section{Methods \\ Cell culture}

Human cell lines SNB19, LN229, LN18, U118MG, T98G, A172, HEK293T, Hela and H460 were obtained from the Cell Bank of the Chinese Academy of Sciences (Shanghai, China). U87 and U251 cells were a gift from Dr. X. Hou (China Pharmaceutical University, Nanjing). HEK293T and $\mathrm{H} 460$ was maintained in RPMI 1640 (Gibco-Invitrogen, CA, USA) supplemented with $10 \%$ fetal bovine serum (FBS, VACCA, Shanghai, China) and penicillin $(100 \mathrm{U} / \mathrm{mL})$-streptomycin $(100 \mu \mathrm{g} / \mathrm{mL})$. All other cell lines were maintained in DMEM (Gibco-Invitrogen) with $10 \%$ FBS and penicillin $(100 \mathrm{U} / \mathrm{mL})$-streptomycin $(100 \mu \mathrm{g} / \mathrm{mL})$. All the above cell lines were authenticated by Biowing Biotech (Shanghai, China).

\section{Antiproliferation assays}

To assess the antiproliferative activity of the targeted agents, cells were seeded in 96-well plates at a density of 1200 (U251) or 2000 (all other cell lines) cells per well and were allowed for growth for $24 \mathrm{~h}$. Subsequently, cells were treated with different concentrations of BKM120, either alone or in combination with different concentrations of another targeted agent. Cellular metabolic activity was assessed after $96 \mathrm{~h}$ treatment by incubation with Alamar Blue solution (Yeasen, Shanghai, China) for another $4 \mathrm{~h}$, following measurement of fluorescence at 534/584 nm using an Fluoroskan Ascent FL analyzer (Thermo Fisher, CA, USA).

\section{Colony formation and 3D spheroids for-mation assay}

To assess the impact of drug treatment on clonogenicity of GBM cells, 400 cells per well were seed-ed in 12-well plates for $24 \mathrm{~h}$ prior to treatment of BKM120, targeted agent, or a combination of both. The drug(s) were added after cell-seeding in the second day, and cells were exposed in the drug-containing medium hereafter with medium refreshing each 3 days. Twelve days after incubation, the colonies were washed with phosphate-buffered saline (PBS) and fixed with 4\% paraformaldehyde for 15 min and stained with a Giemsa Stain solution (Yeasen) 
for $30 \mathrm{~min}$. For the 3D spheroids formation assay, 400 cells per well were seeded in an ultra-low binding 96-well plates with a round bottom (Costar, MA, USA). After 9 days of treatment, digital images of the spheroids were photographed using a phase contrast microscope. The volumes of 3D spheroids were calculated by formula $\left(\mathrm{V}=0.5 *\right.$ Length $\left.{ }^{*} \mathrm{Width}^{2}\right)$ based on the major and minor axial length (Length: major axial length; Width: minor axial length).

\section{Drug combination screening}

A target-selective inhibitors library consisted of 606 target selective small molecule inhibitors (\#L3500) were purchased from Selleck Chemicals. U251 cells were seeded in 96-well plates at a density of at 2000 cells per well $24 \mathrm{~h}$ prior to screening. The drug screen was performed in following steps: First, cells were treated with DMSO (0.2\%), BKM120 $(1 \mu \mathrm{M})$, agent from the drug library $(10 \mu \mathrm{M})$, and combination of BKM120 and targeted agent. SI values were calculated as the formula given in Fig. 2a. As a result, 110 targeted agents exhibited excellent synergies with BKM120 ( $\mathrm{SI} \geq 0.12)$ and were selected for the further validation. Next, the drug sensitivity for U251 of 110 candidate drugs was reassessed as same as the first step and SI was measured again. Finally, six top ranked compounds in the second step were chosen for further assessment of their antiproliferative effects at concentrations of $10 \mu \mathrm{M}$ or $2 \mu \mathrm{M}$ in the presence of $1 \mu \mathrm{M}$ BKM120 across 8 GBM cell lines, and those continuously achieved high SI values were chosen for further investigation.

\section{Western blotting}

Cells were lysed and homogenized in RIPA buffer, and the protein concentrations were determined using a BCA Protein Assay Kit (Thermo Fisher, CA, USA). Protein samples were separated using $10 \%$ sodium dodecyl sulfate-polyacrylamide gels, transferred to PVDF membranes (Merck Millipore, MA, USA) and the membrane was blocked with $3 \%$ bovine serum albumin for $1 \mathrm{~h}$. The blots were incubated with specific primary antibodies for $2 \mathrm{~h}$ at room temperature or $4{ }^{\circ} \mathrm{C}$ overnight, and then incubated with corresponding horseradish peroxidase-conjugated secondary antibodies for $1 \mathrm{~h}$. Finally, it was visualized using an ECL kit (Beyotime, Shanghai, China) and the density of the immunoreactive bands was analyzed using Imager software (Tanon, Shanghai, China). The following primary antibodies used were: pAKT (Ser473) (\#4060), AKT (\#2920), pS6 (Ser235/236) (\#4858), Cleaved-caspase3 (\#9661) (all from CellSignaling, Danvers, USA), p4EBP1 (Ser65) (SC293124) (Santa Cruz Biotechnology, CA), MTH1 (abcam, Cambridge, UK), GAPDH (\#AC002) (ABclonal, Hubei, China).

\section{Comet assay}

Cells were treated with different drugs for $24 \mathrm{~h}$ or $100 \mathrm{mM}$ $\mathrm{H}_{2} \mathrm{O}_{2}$ for 8 min as positive control of DNA damage. Then all cells were harvested, washed and resuspended in PBS at a concentration of $2 \times 10^{6}$ cells $/ \mathrm{ml}$. Next, normal melting agarose was dissolved in the above cell suspension to generate a $0.75 \%$ agarose mixture. $100 \mu \mathrm{l}$ and $450 \mu \mathrm{l}$ cell-agarose mixture were separately added to a fully frosted slide with a $10 \mathrm{~min}$ interval to form two layers in the slide and were kept at $4{ }^{\circ} \mathrm{C}$ for $30 \mathrm{~min}$. After removing the coverslip, the slide was incubated in lysis buffer (10 mM Tris pH 10.0, 2.5 M NaCl, 0.1 M EDTA2Na, 10\% DMSO and $1 \%$ Triton X-100) at $4{ }^{\circ} \mathrm{C}$ overnight. Then the slide was subjected to electrophoresis in Mini Horizontal Cells (BioRad, CA, USA) filled with ice-cold alkaline electrophoresis buffer $(0.3 \mathrm{~N} \mathrm{NaOH}, 1 \mathrm{mM}$ EDTA) at $300 \mathrm{~mA}$ and $25 \mathrm{~V}$ for $30 \mathrm{~min}$. Soaking in neutralization buffer $(0.4 \mathrm{M}$ Tris $-\mathrm{HCl}, \mathrm{pH} 7.5)$ for $5 \mathrm{~min}$, the slide was counter-stained with $5 \mathrm{mg} / \mathrm{ml}$ DAPI (Yeasen). Images were acquired by a Zeiss LSM 510 confocal microscope and were analyzed using a Comet Score software (CASP, CASPLab).

\section{$\mathrm{\gamma}$-H2AX staining}

Cells received treatment for 3 days were harvested and fixed in $75 \%$ ice cold ethanol and kept at $-20{ }^{\circ} \mathrm{C}$ overnight. Cells were spun down and washed with ice-cold PBS containing 2\% FBS for three times. The cell suspension was incubated with an Anti-phospho-histone H2AX (Ser139) (BioLegend Cat. No. 613404, CA, USA) antibody for $1 \mathrm{~h}$ at room temperature. After a brief wash, 10,000 stained cells were collected and analyzed by a BD FACS Verse flow cytometer.

\section{Laser confocal microscope observation}

U251 cells were incubated with TH588 for $48 \mathrm{~h}$ on the glass slide, then washed with PBS for three times, and fixed in $4 \%$ paraformaldehyde about $20 \mathrm{~min}$, washed three times with PBS containing $0.1 \%$ Triton X-100. Then the cells were stained with $\alpha$-tubulin-tracker red (Beyotime Cat. No. C1050) for $60 \mathrm{~min}$ in darkness. A laser scanning confocal microscope (Zeiss LSM880 with NLO \& Airyscan) was used to observe the alterations of cytoskeleton proteins. Excitation and emission wavelength were $488 \mathrm{~nm}$ and $530 \mathrm{~nm}$, respectively.

\section{shRNA construction and MTH1 knockdown}

MTH1 shRNA sequences were designed by an online tool from Sigma-Aldrich website or obtain from literature. Two MTH1 targeting sequences: shRNA\#1 5'-GAA ATTCCACGGGTACTTC-3' and shRNA\#2 5'-CGACGA CAGCTACTGGTTT- $3^{\prime}$ were synthesized and subcloned 
into a pLKO.1-TRC vector via Age1/EcoRI restriction sites. For transfection, the above plasmids, empty vector together with the packaging plasmids pVSVg (\#8454, Addgene) and psPAX2 (\#12260, Addgene) were transfected into HEK293T cells at approximately $60 \%$ confluence using polyethylenimine (PEI) for $48 \mathrm{~h}$. The culture medium of U251 cells was replaced with the above lentivirus-containing supernatants with the addition of 1:1000 of polybrene and was centrifuged for $2 \mathrm{~h}$ at $37^{\circ} \mathrm{C}$ for viral infection. After puromycin selection for 3-7 days, the infected cells were collected for validation of MTH1 knockdown.

\section{Cell apoptosis assay by flow cytometry}

Cells received drug treatment for $24 \mathrm{~h}$ were collected, washed with ice-cold PBS, and evaluated for apoptosis by double-staining with FITC and PI with an Apoptosis Detection Kit (Yeasen Biotech). Stained cells were analyzed with a FACS Calibur flow cytometer and the obtained data were analyzed by FlowJo 7.6.

\section{Analysis of PTEN expression-drug sensitivity interactions from public database}

We took advantage of the Wooster cell line dataset which consists of over 300 cell lines from 19 cancer types (also see https://cabig.nci.nih.gov/caArray_GSKdata/) for analysis of the correlation between PTEN expression and their sensitivities to PI3K inhibitors. All cell lines were divided into three groups: resistant, intermediate sensitive and sensitive according to their IC50 upon treatment of BKM120 or GSK1059615. The PTEN expressions of all cell lines were obtained from Oncomine (https://www. oncomine.org/).

\section{Statistics}

All experiments except the drug screen and the Western Blotting were performed at least three times independently. Western blotting experiments were performed at least twice independently. The data are expressed as the mean \pm SD. Statistical analyses were performed with GraphPad Prism 5.0 software (San Diego, CA, USA). The isobologram analysis of the two drug combinations was performed using CompuSyn 2.0 software (ComboSyn, Inc., Paramus, NJ, USA) [18]. Statistical significance was determined using the two-tailed Student's $t$ test unless otherwise mentioned, with the following $P$ values considered significant: ${ }^{*} \mathrm{P}<0.05$; ${ }^{* * \mathrm{P}}<0.01$; ***: $\mathrm{P}<0.001$.

\section{Results}

BKM120 blocked PI3K-AKT signaling and exhibited cell line-dependent anti-glioma effects

We first investigated the antiproliferative effect of BKM120 using cell viability and colony formation assays across eight GBM cell lines. BKM120 exhibited general growth inhibitory effects in a dose-dependent manner, but limited responsiveness was observed for several cell lines, such as U251, compared with sensitive cell lines like U87 or T98G (Fig. 1a, b). Next, we selected BKM120 sensitive and insensitive cell lines for further investigation of signaling pathway perturbation. Exposure of U251, U87 and T98G cells to BKM120 resulted in suppression of AKT and $\mathrm{S} 6$ phosphorylation in a dose-dependent manner, suggesting that the PI3K-AKT signaling was sufficiently blocked even in the BKM120 insensitive cell line (Fig. 1c).

PTEN deletion or mutation is a critical event frequently occurred during GBM development. More than half of the GBM cell lines harbored mutations in the PTEN gene (Additional file 1: Figure S1A), and this could affect the activities of PI3K inhibitor as an anticancer agent [19]. We took advantage of the PTEN WT and $\mathrm{KO}$ isogenic LN18 cell lines that our lab established previously to investigate the relationship between the PTEN status and the anti-glioma effect of BKM120. As shown in Additional file 1: Figure S1B, PTEN loss did not confer sensitivity to BKM120 in LN18 cells. Furthermore, we analyzed the Wooster cell line dataset which comprise over 300 cell lines treated with 19 drugs. Again, whether these cell lines were sensitive or resistant to BEZ235 or GSK1059615, another pan PI3K inhibitor, were independent of the levels of PTEN expression (Additional file 1: Figure S1C). We also calculated Spearman's correlation between PTEN protein expression (data obtain from the cell line reverse-phase protein array in the Cancer Cell Line Encyclopedia project) and IC50 (obtained from the Genomics of Drug Sensitivity in Cancer project). We found that low PTEN expression even confers marked increased resistance instead of sensitivity to BKM120 treatment, for a negative $(\mathrm{P}<0.05)$ correlation between the expression of PTEN and the IC50 of another PI3K inhibitor AS605240 was observed (Additional file 1: Figure S1D).

\section{Drug combination screening identified compounds synergizing anti-glioma effect with BKM120}

Evaluation of the drug combination effect can be carried out via various methods and models. However, most of them are based on a dose response matrix, e.g., the Highest Single Agent (HSA) and Bliss independence models [20-22], but such approaches are laborious when screening hundreds of drugs. To simplify this process, we established a method for high-throughput drug combination screening in which a Sensitivity Index (SI) score was introduced to quantify the influence of the addition of another drug. SI was defined as the difference between observed combined effect and expected 


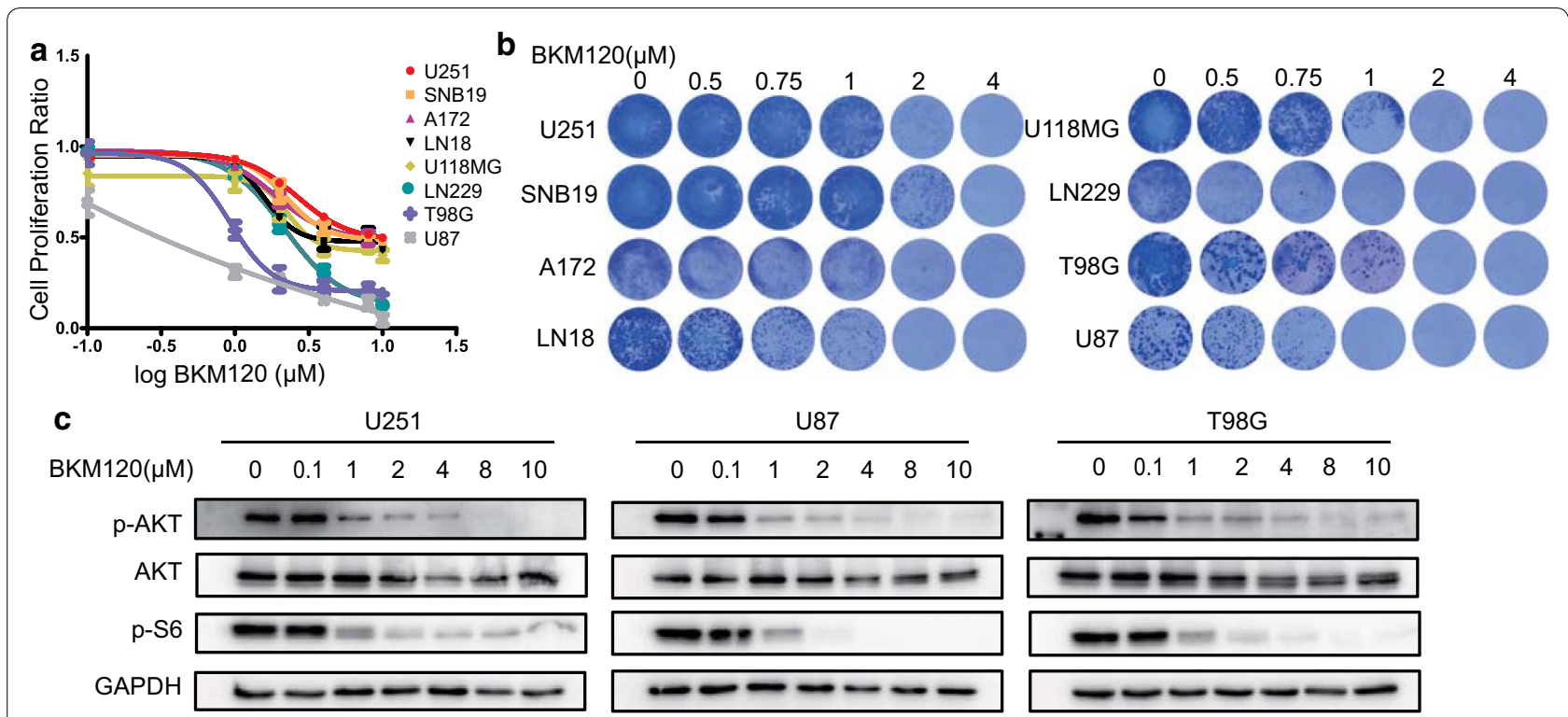

Fig. 1 Evaluation of the anti-glioma effect of single agent BKM120. a The antiproliferative effect of BKM120 as single agent treatment in eight GBM cell lines. Cell viability was measured with Alamar Blue. Data are presented as percentages relative to the vehicle control. $\mathbf{b}$ Images of colonies formed by eight GBM cell lines incubated with different concentrations of BKM120 for 14 days followed by Giemsa stain solution on the last day of incubation. c Western blot analysis showing blockage of PI3K pathway signaling by BKM120 in three cell lines. Three GBM cell lines were incubated with different concentrations of BKM120 for $24 \mathrm{~h}$

combined effect which is calculated as the product of the relative proliferation ratio of cells treated with BKM120 and a certain library drug (Fig. 2a). The expected combined effect is estimated as a minimal additive effect of two drug because it is based on an assumption that cells only received BKM120 and a library drug sequentially and no residual effect of BKM120 could affect the following treatment. This formula was also used for calculation of the influence of siRNA-induced knockdown of gene expression on drug sensitivity [23]. The SI scores ranged from -1 to +1 , with positive values indicating BKM120sensitizing effects.

From the above results, we determined that $1 \mu \mathrm{M}$ BKM120 was an appropriate concentration for the combination drug screen, and the U251 cell line was chosen for its insensitivity to BKM120. We took advantage of a drug library containing 606 highly selective small molecule inhibitors targeting multiple cancer-related signaling pathways (Additional file 2: Figure S2). To calculate the SI for individual drugs in the drug library, U251 cells received the following treatments: vehicle (DMSO), $1 \mu \mathrm{M}$ BKM120, $10 \mu \mathrm{M}$ library drug and a combination of $1 \mu \mathrm{M}$ BKM120 and $10 \mu \mathrm{M}$ library drug (Fig. 2b).

The first step resulted in the identification of 110/606 (1.8\%) inhibitors with $\mathrm{SI} \geq 0.12$, a threshold at which potential BKM120-sensitizing effects were expected. In the second step, the antiproliferative activity of these inhibitors was then re-assessed at $10 \mu \mathrm{M}$ on $\mathrm{U} 251 \pm 1 \mu \mathrm{M}$
BKM120 to confirm the candidates identified in the first step. Finally, the 6 top ranked compounds displaying relatively strong synergistic effects with BKM120 in the second step were chosen for further assessment of their antiproliferative effects at $10 \mu \mathrm{M}$ and $2 \mu \mathrm{M}$ in the presence of $1 \mu \mathrm{M}$ BKM120 across 8 GBM cell lines (Fig. 2b).

\section{Validation of the synergism between BKM120 and the candidate agents}

For the six top candidates selected above, SI were reassessed to ensure their abilities to sensitize cells to the antiproliferative effect of BKM120. All candidate compounds had a BKM120-sensitizing effect $(\mathrm{SI}>0)$, and five of them exhibited a strong sensitizing effect $(\mathrm{SI}>1.0)$ (Fig. 3a). Next, we assessed the antiproliferative effect of the combination treatment of eight GBM cell lines. Although differentiated responses were observed across different cell lines and across different treated concentrations, the mean SI of all compounds were all above 0 , suggesting an overall BKM120-sensitizing effect of these candidates. A brief summary of the top six candidates were given (Fig. 3b). Finally, the top four candidate compounds, TH588 (an MTH1 inhibitor), CGP57380 (an MNK1 inhibitor), GW9662 (a PPAR inhibitor) and 4-hydroxytamoxifen (an estrogen receptor inhibitor), were chosen for further evaluation. As shown in colony formation assays, these compounds almost eliminated all colonies of U251 in the presence of BKM120, while a single drug or BKM120 


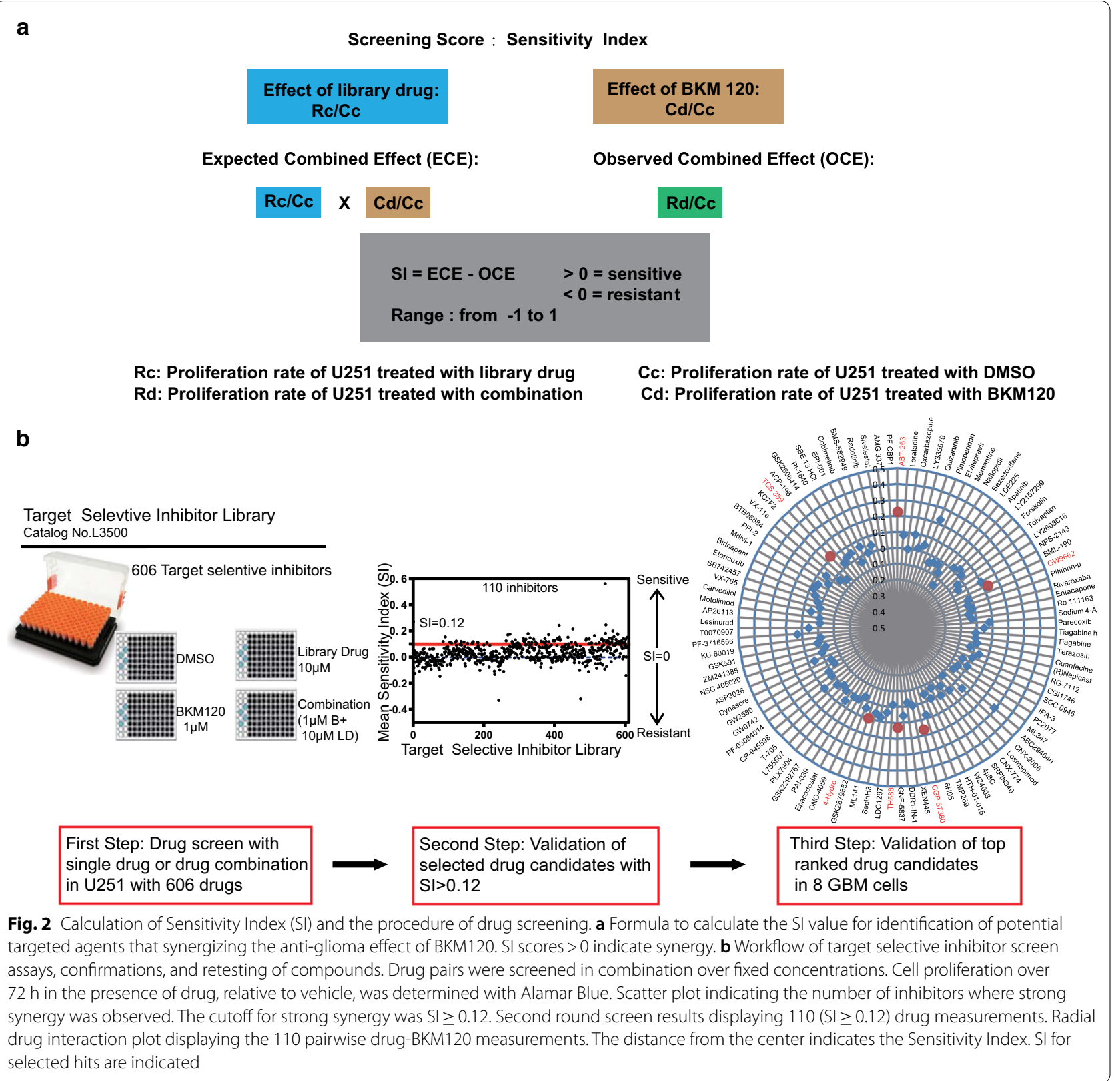

failed to show such a strong effect (Fig. 3c). Among them, ABT263, CGP57380 and 4-hydroxytamoxifen have already been reported for their synergistic effects in combination with BKM120 [24-26]. Altogether, this evidence supports that our proof-of-principle screening method is useful to identify potential drug combinations that have synergistic interactions with BKM120.

\section{Characterization of the anti-glioma effect of the combination treatment of BKM 120 and TH588}

Because TH588 was the top candidate and has not yet been reported elsewhere for its combined effect with
BKM120, it was chosen for further investigation. U251 and SNB19 cells were treated with a 5-by-5 dose titration matrix of TH588 and BKM120, and the viability of the cells was determined. As shown in Fig. 4a, d, the expected versus observed dose-response surface plot of the 5-by-5 matrix viability data demonstrated synergistic effect of two agents against U251 and SNB19 GBM cells. In addition, the combination treatment led to marked reduced viability of GBM cells. Moreover, most dots (each dot represents a combination of a specific concentration of two agents) had a CI value below 1.0 in the Fa-CI plots, suggesting the interaction between TH588 and BKM120 


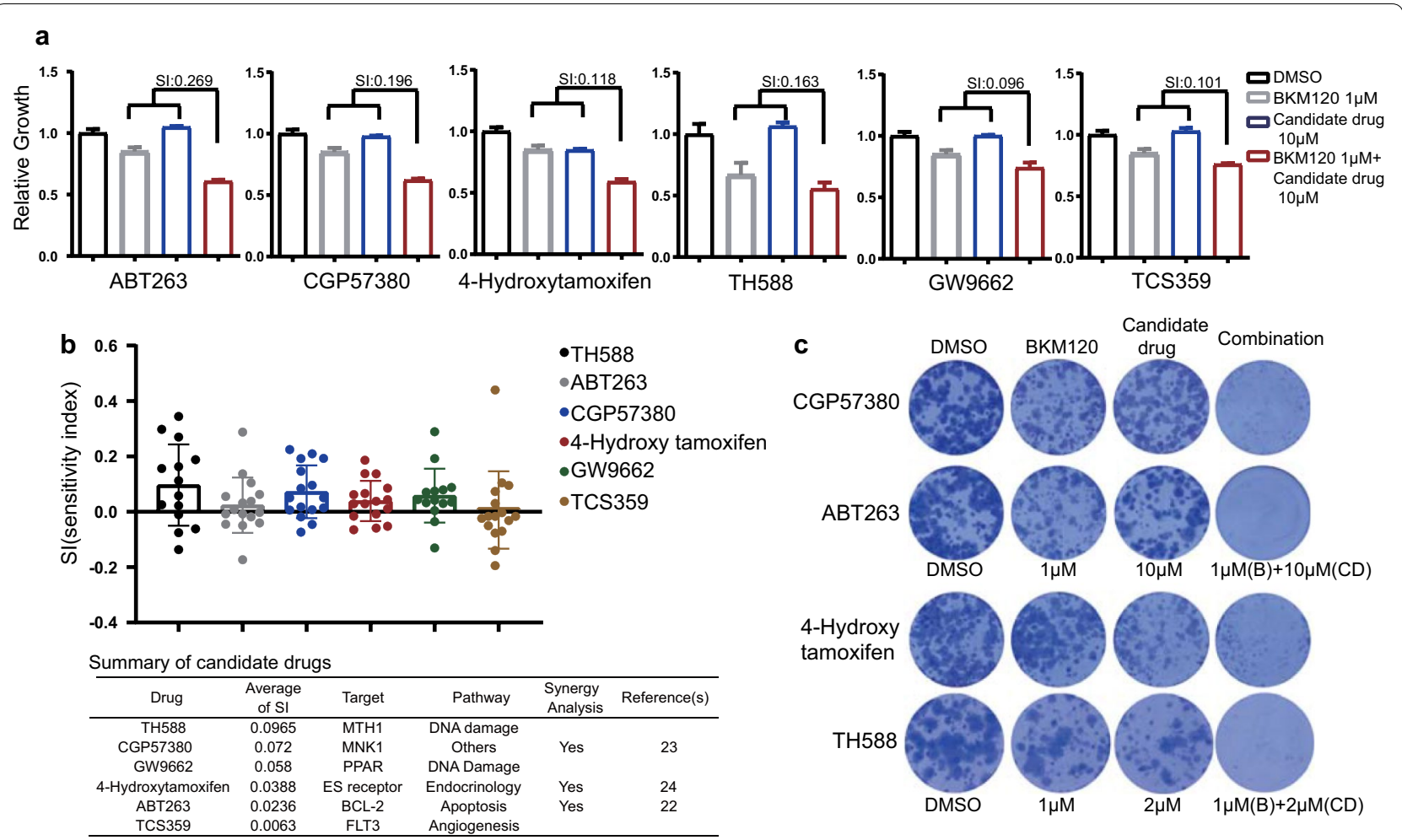

Fig. 3 Validation of the BKM120 synergizing effects of candidate agents. a Cell viability of U251 cells upon treatment with BKM120 in the presence and absence of a fixed concentration of 6 candidate compounds. $\mathbf{b}$ Distribution of SI values of multiple GBM cell lines received treatment of BKM120 and targeted agents at different concentrations $(2 \mu \mathrm{M}, 10 \mu \mathrm{M})$ and a summary of top ranked 6 targeted agents $\mathbf{c}$ Images of colonies formed by $\mathrm{U} 251$ cells incubated with library inhibitors \pm BKM120 for 14 days and Giemsa stained on the last day of incubation

were synergistic (Fig. 4b, e). We also applied classical synergy models including HSA and Bliss [20-22] to determine the HSA and Bliss values, which are a readout for synergistic inhibition and depict the difference between expected inhibition and observed inhibition. Most of the HSA and Bliss values were above 0, indicating synergistic interactions between the two drugs (Fig. 4c, f). Furthermore, the combination of TH588 and BKM120 also severely suppressed colony formation in eight GBM cell lines (Fig. 4g) and 3D spheroid formation in SNB19 and LN229 cells (Fig. 4h-k), confirming that the effect of the combination of the two drugs exceeds these of each single agent in suppressing the growth and proliferation of GBM cells in different models.

\section{The combination of BKM120 and TH588 synergistically induces oxidative DNA damage and apoptosis}

TH588 has been reported to cause DNA damage by increasing 8-oxodG or 2-OH-dA incorporation into DNA, leading to DNA base mispairing, mutation and cell death [27]. Interestingly, previous works suggest that inhibition of PI3K interferes with DNA synthesis or repair through nucleoside depletion [28] so there might be a link between BKM120 and TH588. To reveal the synergistic mechanism, we used a comet assay, a sensitive method for detecting 8-oxodG or 2-OH-dA at the single-cell level to evaluate the DNA damage caused by the combination treatment [29]. The significant elongated tailing ratio of combination treatment relative to control or single agent treatment suggests that more abundant DNA-damaged fragments and accumulation of 8-oxodG in DNA were presented after treatment of both MTH1 and PI3K inhibitor (Fig. 5a, b).

The nucleosomal histone protein $\mathrm{H} 2 \mathrm{AX}$ is rapidly phosphorylated at serine $139(\gamma-\mathrm{H} 2 \mathrm{AX})$ at the double strand break (DSB) site [30], so $\gamma-\mathrm{H} 2 \mathrm{AX}$ foci formation is usually used to quantify DSBs. Therefore, we reasoned that the PI3K inhibitor also enhanced the DSB damage of DNA caused by TH588. To confirm that, GBM cells treated with BKM120 and/or TH588 was stained with $\gamma-\mathrm{H} 2 \mathrm{AX}$ and analyzed by flow cytometry. As shown in Fig. 5c, d and Additional file 3: Figure S3, the $\gamma-\mathrm{H} 2 \mathrm{AX}-$ positive fraction of U251 cells increased significantly upon treatment with BKM120 and TH588 compared with that in cells that received single-agent treatment, suggesting that the combination of PI3K and MTH1 


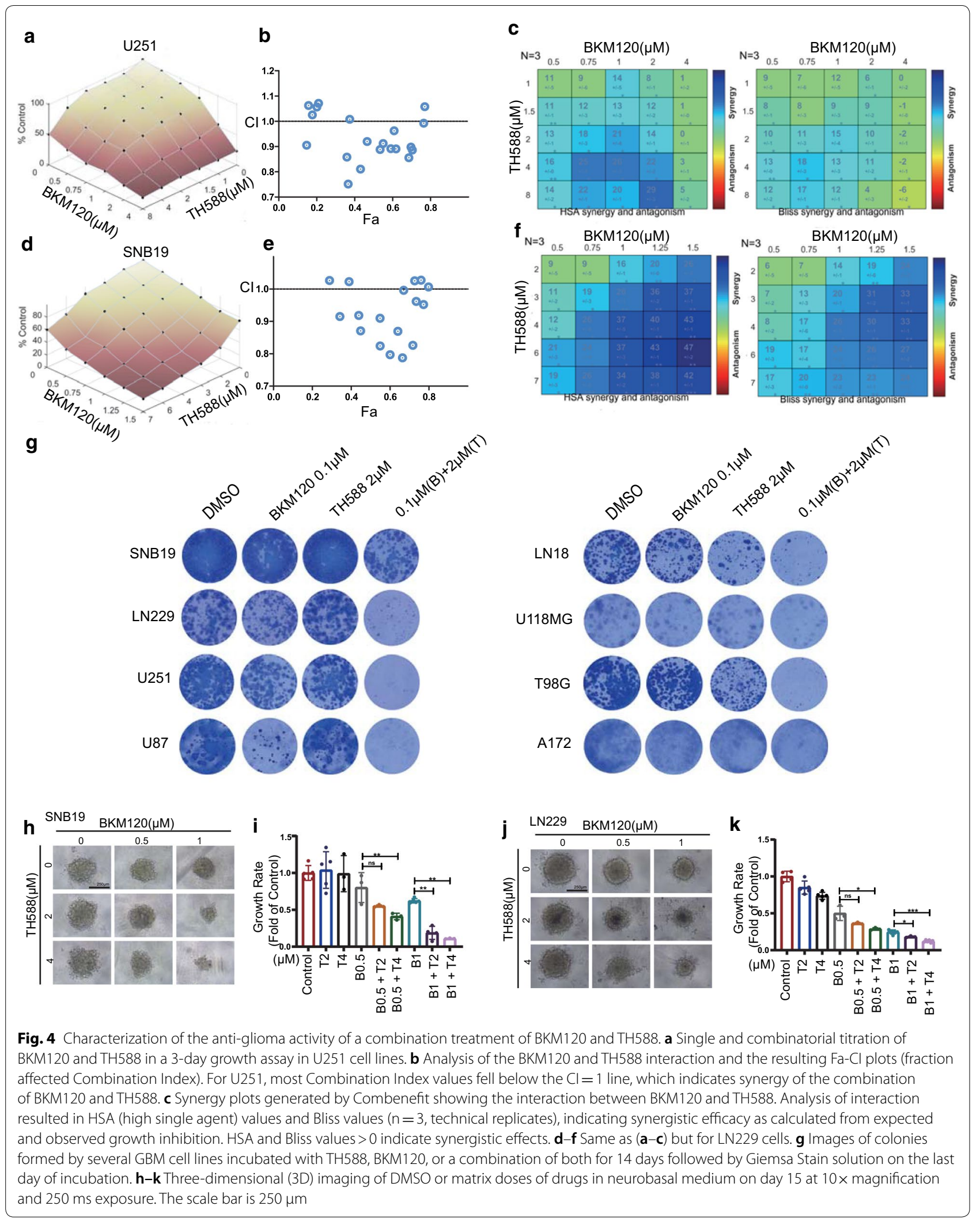




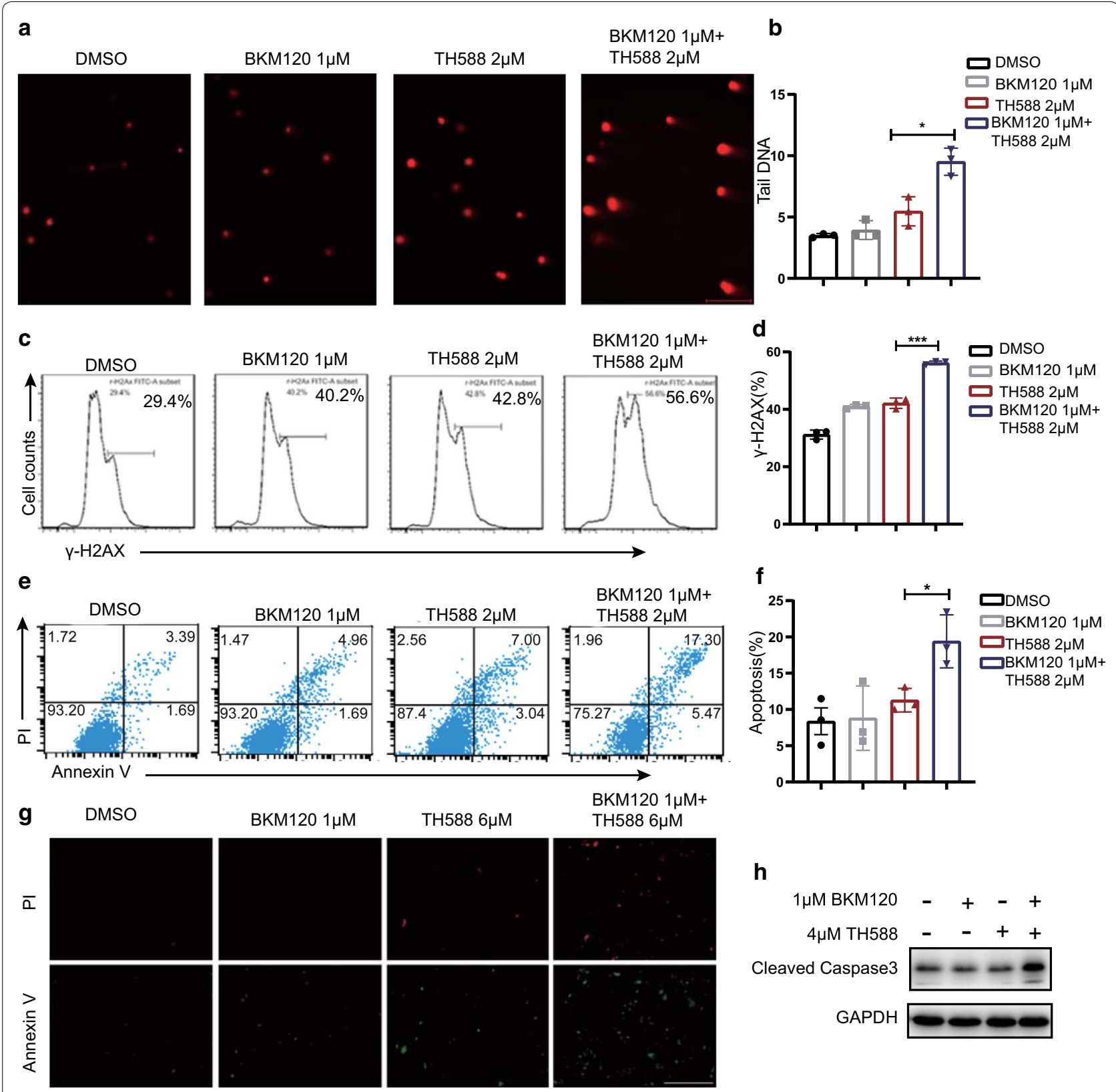

Fig. 5 The combination of BKM120 and TH588 synergistically induces oxidative DNA damage and apoptosis. a Comet assay of U251 cells following treatment with BKM120, TH588 or the combination for $24 \mathrm{~h}$. H2O2 was used as a control. b Quantification of comet tail moment. Tail moment, calculated as \% DNA in the tail multiplied by the tail length. Values represent the average \pm SD from three independent experiments ( $>25$ comets per experiment, $\left.{ }^{* *} \mathrm{P}<0.01\right)$. c Flow cytometry assay of $\mathrm{H} 2 \mathrm{AX}$ phosphorylation in $\mathrm{U} 251$ cells following treatment with increasing concentrations of TH588 for 24 h. d Quantification of $\mathrm{Y}$-H2AX-positive cells from $\mathrm{B}\left({ }^{*} \mathrm{P}<0.05,{ }^{* *} \mathrm{P}<0.01\right)$. e Flow cytometric analysis of \% apoptotic U251 cells (stained by annexin V/PI) following BKM120, TH588 or combination pretreatment for $24 \mathrm{~h}$. $\mathbf{f}$ Quantification of apoptotic cells from $\mathbf{f}$; shown is the average $\pm S D, n=3\left({ }^{*} P<0.05\right.$, $\left.{ }^{* *} P<0.01\right) . \mathbf{g} \cup 251$ cells treated with BKM120, TH588 or a combination of both for $48 \mathrm{~h}$, and then subjected with co-staining with annexin $\mathrm{V}$ and PI. Cells were observed under a fluorescent microscope and representative photos were given. Scale bars $=0.2 \mathrm{~mm}$. h $U 251$ cells received treatment of BKM120,TH588 or a combination of both for 48 h were immunoblotted with antibodies of cleaved caspase-3 and GAPDH

inhibitors dramatically elevates DSB levels in GBM or lung cancer cells. Accumulating evidence suggests that PI3K pathway is also involved in DNA replication and genome stability [28]. Therefore, PI3K might be an attractive target for combining with targeted agent related to DNA repairing pathway [31]. 
Moreover, comparing with the independent treatment of BKM120 and TH588, the combination treatment significantly increased the Annexin V-positive apoptotic fraction of cells (Fig. 5e-g and Additional file 5: Figure S5), and also caused marked elevation of cleaved caspase3.

On top of the MTH1 inhibition, alternative mechanisms of TH588 have been reported as its anti-cancer effects including tubulin depolymerization and AKT signaling downregulation [32, 33]. To confirm it, we investigated the spindle morphology of mitotic cells upon treatment of 5 and $10 \mu \mathrm{M}$ of TH588. Indeed, TH588 caused disruption of spindle formation and interrupted the separations of duplicated centrosomes (Additional file 3: Figure S3). In addition, treatment of TH588 also caused reduction of phosphor Akt (S473) and its downstream component 4EBP1 (S65) in U251 and U87 cells (Additional file 3: Figure S3). Together these evidence provide additional information for TH588 mediated GBM cell death besides MTH1 inhibition.

\section{The PI3K/AKT signaling is a determinant of the responsiveness to MTH 1 inhibition}

To assure that the antiproliferative activity of TH588 is due to its selective targeting of MTH1 but not an off-target effect, stable MTH1 knockdown U251 together with control cells transduced with only empty vector control were established. The expression of MTH1 in two MTH1 knockdown cell lines was validated by Western blotting (Fig. 6a). As shown in Fig. 6b, MTH1-silenced U251 cells were generally sensitive to BKM120, and the IC50 of two MTH1-silenced cells (2.59 and 1.63) were lower than that of control cells (2.99).

To further investigate whether the TH588 synergizing effect with PI3K inhibitor only limit to BKM120, we also assessed the combined anti-glioma effect of TH588 with other PI3K and AKT inhibitors. For GDC-0941, another pan-PI3K inhibitor, it effectively suppressed the proliferation of GBM cells with TH588, and the Combination Index $(\mathrm{CI})$ values were lower than 1 at most concentrations, suggesting the interaction between two agents were synergistic (Fig. 6c). For GDC-0068 and MK-2206, two highly selective AKT inhibitors, most $\mathrm{CI}$ values were also below 1 when they were combined with TH588 (Fig. 6d, e). The above evidence suggested that both PI3K and AKT inhibitors enhanced the antiproliferation effect of MTH1 blockade or knockdown, indicating that the PI3KAKT pathway activation may be an important determinant for the anti-glioma efficacy of MTH1 inhibition.

To find more evidence supporting this finding, we also checked the levels of PI3K-AKT signaling activation in eight GBM cell lines and categorized them into low, medium or high groups according to the expression levels of p-AKT (Fig. 6f). Next, we performed a proliferation assay by treating these GBM cell lines with TH588 and analyzing the corresponding IC50. Although the number of cell lines used was limited, GBM cells with highly activated PI3K-AKT signaling tended to be more resistant to TH588 (IC50 values for the p-AKT low, medium and high groups were $>14.56,9.19$ and $8.98 \mu \mathrm{M}$, respectively, Fig. 6g). Based on this observation, we established LN229 isogenic cell lines with PTEN wild type, PTEN knockout, and PIK3CA carrying constitutively activated mutations (p.N345K and p.E542K) [34] and investigated their responses to MTH1 inhibition. It is known that lossof-function mutations of PTEN and gain-of-function mutations of PIK3CA can both activate the PI3K-AKT pathway. Indeed, the levels of p-AKT and its downstream component p-S6 were elevated in all three mutant cell lines compared with LN229 parental cells (Fig. 6h). In line with the above results, both PTEN knockout and PIK3CA mutant cells were less responsive to TH588 than their parental cell lines (Fig. 6i). Finally, we transfected a plasmid carrying the intact AKT gene or an empty vector into HeLa cells, and it led to elevation of p-AKT, p-S6 and $\mathrm{p}-4 \mathrm{EBP} 1$ expression in AKT-overexpressing cells relative to empty vector-transfected cells (Fig. 6j). Similarly, AKT-overexpressing cells showed significantly elevated TH588 tolerance compared with that in empty vectortransfected control cells (Fig. 6k). Altogether, we found that the anti-glioma effect of TH588 was determined by the activation of the PI3K-AKT signaling pathway, which may provide an explanation for the synergistic interaction between PI3K and MTH1 inhibitors.

\section{Discussion}

In this study, we developed a simple and efficient screening method for high-throughput identification of agents that synergize with a PI3K inhibitor. The primary screening was based on the Sensitivity Index, which was calculated as the difference between the observed combined effect and expected combined effect. As a result, at least six promising BKM120 synergistic combinations were identified and validated. Among them, three top-ranked candidates, namely, MNK1, estrogen receptor and BCL-2 inhibitors, have been reported previously for their antitumor effects in combination with PI3K/mTOR inhibitors, confirming the usefulness of this screening method. Importantly, in this study, we successfully identified a novel combination of BKM120 and the MTH1 inhibitor TH588, which had a potent anti-glioma effect. Further investigation suggested that the synergy between PI3K and MTH1 inhibitors was not only limited to BKM120 and TH588, but also can be extended to AKT or other PI3K inhibitors together with TH588. 


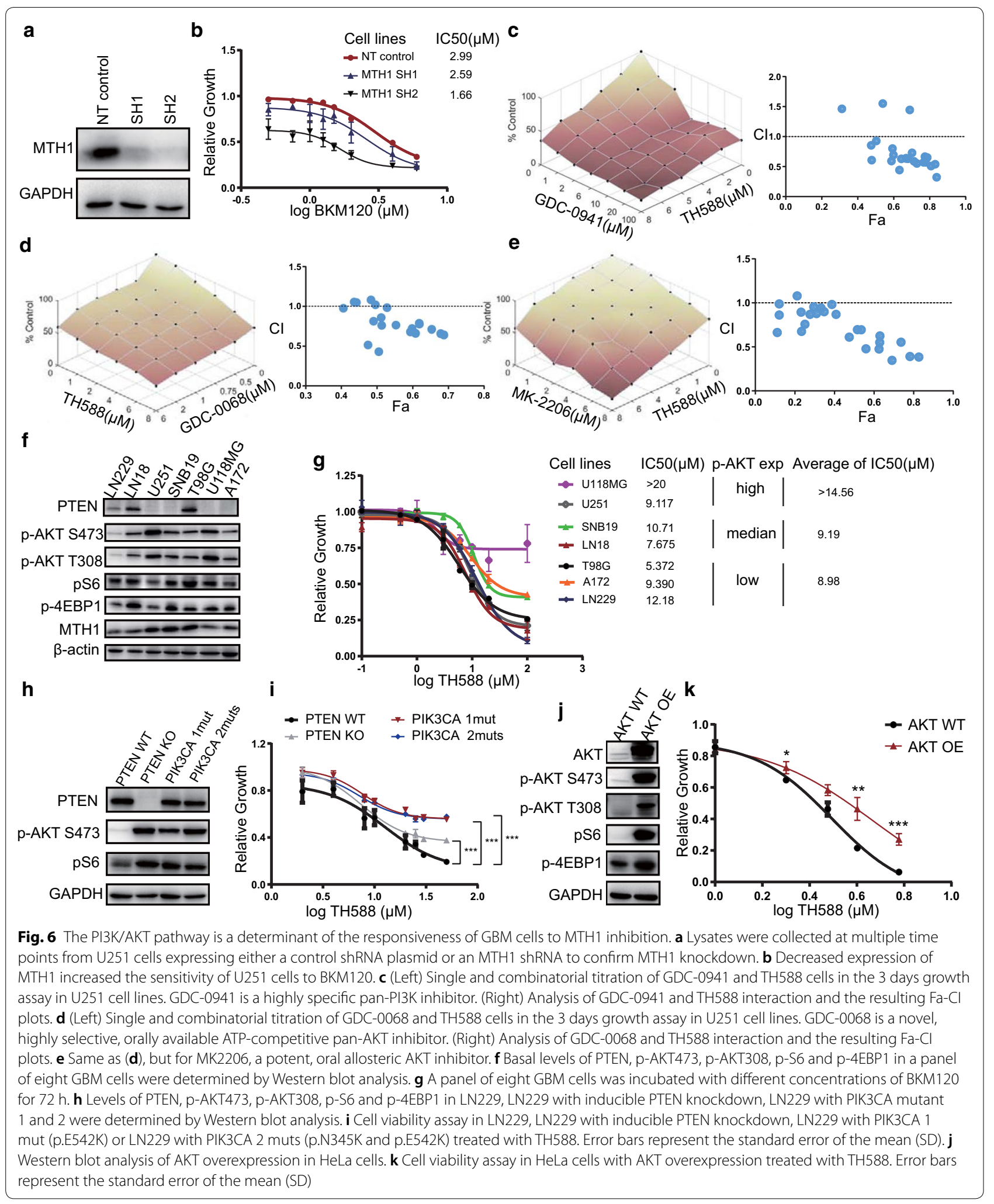


Single-agent PI3K inhibitors failed to exhibit a significant therapeutic response in clinical trials of malignant gliomas for multiple reasons. The data presented here showed that BKM120 alone suppressed the PI3K-AKT pathway as well as arrested GBM cell proliferation, but this effect was dose-dependent and cell line-dependent. In addition, GBMs carrying PTEN mutations or deletions were previously thought to be sensitive to PI3K inhibitors. However, we failed to find that these tumors responded better to PI3K inhibitors than PTEN wildtype GBM. Given the importance of the PI3K-AKT signaling pathway and the fact that PTEN was mutated or deleted in $40-60 \%$ of GBMs, it is necessary to explore effective combined treatment to boost the anti-glioma effect of PI3K inhibitors. As a result of the drug screening, six compounds that significantly enhanced the antiglioma effect of BKM120 in the PTEN-deficient GBM cell line were successfully identified, and the MTH1 inhibitor TH588, which had the highest SI score in the screen, was further studied.

The human mutT homologue MTH1 is a human 8-oxo-dGTPase that removes oxidized bases in the nucleotide pool and DNA, thus avoiding ROS-induced DNA misincorporation, mutations and cell death [27]. In contrast, normal cells repair oxidative damage to DNA via alternative pathways. The severe dependence on MTH1-mediated DNA repair in cancer cells makes MTH1 an attractive target in cancer treatment $[35,36]$. Recent reports indicated that cancer cells were also heavily dependent on functional MTH1 to maintain stemness and tumorigenesis [37, 38]. Several small molecular inhibitor of MTH1 including TH588, BAY707 and S-crizotinib were developed and their anti-tumor activities looks promising $[27,39,40]$. For TH588, some researchers questioned its target specificity as this agent also possesses microtubule-modulating activity. Indeed, in our study we also found that treatment of TH588 cause mitotic spindles disruption beside its potent anti-GBM effect in the presence of BKM120. Therefore, TH588 may has other target(s) except MTH1. Interestingly, previous work reported the effectiveness of the combination of TH588 and everolimus, an mTORC1 inhibitor, in the treatment of neuroendocrine cancer cells [32]. However, brain delivery across the blood-brain barrier of everolimus is limited [7]. In this study, we reported the synergy between TH588 and BKM120, a pan-PI3K inhibitor penetrating the blood-brain barrier more efficiently than rapalogs [41].

Some evidence from previous works supports our finding that there are functional interactions between PI3K pathway inhibition and ROS-induced DNA damage repair $[27,28]$ and PARP-mediated base excision repair [42]. Indeed, we found a significant increase in the proportion of segmented tail DNA and phosphorylated H2AX under combined treatment with TH588 and BKM120, suggesting that the DNA damage repair pathway was indeed affected by the PI3K pathway and that blockade of both causes severe consequences for cancer cells.

\section{Conclusions}

In conclusion, with a simple and efficient screening method, we successfully identified several promising and effective combinations of targeted drugs with BKM120 that potently inhibit GBM proliferation and growth. Among them, an MTH1 inhibitor TH588 excelled other top ranked candidate targeted agents and exhibited a strong synergistic anti-glioma effect with BKM120, thus offering a potential combination therapy for further evaluation in GBM models. Overall, the screening method used in this study provides a useful and reliable tool for finding novel and effective combination therapies for refractory tumors such as GBM.

\section{Supplementary information}

Supplementary information accompanies this paper at https://doi. org/10.1186/s12935-020-01427-0.

Additional file 1: Figure S1. PTEN gene mutations in GBM cells and its impact on drug sensitivities of PI3K inhibitors. (A) Mutations of four essential GBM oncogenes or tumor suppressor genes in eight GBM cell lines. (B) Antiproliferation rate of LN18, LN18 sgRNA negative control, LN18 PTEN knockout \#1 and 2 cell lines upon treatment of BKM120. (C) PTEN expression in PI3K inhibitor sensitive, intermediate sensitive or resistant cell lines collection. GSK1059615 is a dual inhibitor of pan-class I PI3K and mTOR kinase. (D) Linear regression of PTEN protein levels and BKM120 sensitivity demonstrated a strong correlative trend in terms of $R$ and $p$ values by Spearman's rank correlation test $(p=0.0256)$. Data of $C$ and $D$ were obtained from public database (Wooster dataset and Oncomine database).

Additional file 2: Figure S2. Summary of the targeting pathways of 606 small molecule inhibitors in the drug library (Selleck \#L3500).

Additional file 3: Figure S3. Treatment of BKM120 and TH588 caused elevation of $\mathrm{\gamma}-\mathrm{H} 2 \mathrm{AX}$-positive cells. Left: Flow cytometry analysis of $\mathrm{\gamma}-\mathrm{H} 2 \mathrm{AX}$ stained LN229 GBM cells following treatment of vehicle (DMSO), BKM120, TH588 and combination of both for $24 \mathrm{~h}$. Right: Quantification of $\mathrm{Y}-\mathrm{H} 2 \mathrm{AX}$ positive LN229 cells of each type of treatment in triplicates.

Additional file 4: Figure S4. Flow cytometric analysis of apoptotic cells upon treatment of TH588 and/or BKM120. Left: H460 cells were treated with vehicle (DMSO), BKM120, TH588 or combination of both for $24 \mathrm{~h}$ and analyzed by flow cytometry for quantification of the fraction of apoptotic cells (pre-stained with annexin V/PI). Right: Quantification of apoptotic fraction of $\mathrm{H} 460$ cells received each type of treatment in triplicates.

Additional file 5: Figure S5. TH588 disrupts mitotic spindles and causes AKT pathway downregulation. (A) Photomicrographs of mitotic cells treated with DMSO or TH588 for 48 hours showing a-tubulin (red), and chromatin (blue, DAPI). Scale bar $=10 \mu \mathrm{m}$. (B) Western blot analysis of components from the AKT pathway were analyzed after $48 \mathrm{~h}$ treatment of TH588.

Abbreviations

GBM: Glioblastoma multiforme; SI: Sensitivity index; TCGA: The cancer genome atlas; CCLE: Cancer cell line encyclopedia; GDSC: Genomics of drug sensitivity 
in cancer; IC50: Half maximal inhibitory concentration; Cl: Combination index; HSA: Highest single agent; BLISS: Bi-level intergrated system synthesis; DSB: Double strand breaks; DMSO: Dimethyl sulfoxide; PBS: Phosphatebuffered saline.

\section{Acknowledgements}

Not applicable.

\section{Authors' contributions}

Conception and design: FL Development of methodology: FL, ZC, YH. Acquisition of data: ZC, TZ, CC, QW, CD, XZ, XZ, FW. Analysis and interpretation of data: ZC, TZ, CC, FL. Writing, review of the manuscript: FL, ZC. All authors read and approved the final manuscript.

\section{Funding}

This study was supported by the National Natural Science Foundation of China (81672962), the Jiangsu Provincial Innovation Team Program Foundation, and the Joint Key Project Foundation of Southeast University and Nanjing Medical University.

\section{Availability of data and materials}

The analysed data sets generated during the study are available from the corresponding author on reasonable request.

\section{Consent for publication}

The content of this manuscript has not been previously published and is not under consideration for publication elsewhere and it has been read and approved by all the co-authors.

\section{Competing interests}

The authors declare no conflicts to disclose.

\section{Author details}

1 Department of Cell Biology, School of Basic Medical Sciences, Nanjing Medical University, XueHai Building A111, 101 Longmian Avenue, Nanjing, Jiangning District, China. ${ }^{2}$ Department of Oncology, The First Affiliated Hospital of Nanjing Medical University, Nanjing, China. ${ }^{3}$ College of Pharmaceutical Science, Zhejiang University of Technology, Hangzhou, China. ${ }^{4}$ Institute for Brain Tumors, Key Laboratory of Rare Metabolic Diseases, The Affiliated Cancer Hospital of Nanjing Medical University; Key Laboratory of Human Functional Genomics of Jiangsu Province, Nanjing, China.

Received: 28 April 2020 Accepted: 15 July 2020

Published online: 23 July 2020

\section{References}

1. Weller M, van den Bent M, Hopkins K, Tonn JC, Stupp R, Falini A, et al. EANO guideline for the diagnosis and treatment of anaplastic gliomas and glioblastoma. Lancet Oncol. 2014;15(9):e395-403.

2. Stupp R, Mason WP, van den Bent MJ, Weller M, Fisher B, Taphoorn MJ, et al. Radiotherapy plus concomitant and adjuvant temozolomide for glioblastoma. New Engl J Med. 2005;352(10):987-96.

3. Shergalis A, Bankhead A 3rd, Luesakul U, Muangsin N, Neamati N. Current challenges and opportunities in treating glioblastoma. Pharmacol Rev. 2018;70(3):412-45.

4. Engelman JA, Luo J, Cantley LC. The evolution of phosphatidylinositol 3-kinases as regulators of growth and metabolism. Nat Rev Genet. 2006;7(8):606-19.

5. Cancer Genome Atlas Research N. Comprehensive genomic characterization defines human glioblastoma genes and core pathways. Nature. 2008;455(7216):1061-8.

6. Vivanco I, Sawyers CL. The phosphatidylinositol 3-Kinase AKT pathway in human cancer. Nat Rev Cancer. 2002;2(7):489-501.

7. Kreisl TN, Lassman AB, Mischel PS, Rosen N, Scher HI, Teruya-Feldstein J, et al. A pilot study of everolimus and gefitinib in the treatment of recurrent glioblastoma (GBM). J Neurooncol. 2009;92(1):99-105.

8. Chang SM, Wen P, Cloughesy T, Greenberg H, Schiff D, Conrad C, et al. Phase II study of CCl-779 in patients with recurrent glioblastoma multiforme. Invest New Drugs. 2005;23(4):357-61.
9. Greenwell IB, Ip A, Cohen JB. PI3K inhibitors: understanding toxicity mechanisms and management. Oncology. 2017;31(11):821-8.

10. Engelman JA. Targeting PI3K signalling in cancer: opportunities, challenges and limitations. Nat Rev Cancer. 2009;9(8):550-62.

11. Muranen T, Selfors LM, Worster DT, I wanicki MP, Song L, Morales FC, et al. Inhibition of PI3K/mTOR leads to adaptive resistance in matrix-attached cancer cells. Cancer Cell. 2012;21 (2):227-39.

12. Cloughesy TF, Mischel PS. New strategies in the molecular targeting of glioblastoma: how do you hit a moving target? Clin Cancer Res. 2011;17(1):6-11.

13. Lehar J, Krueger AS, Avery W, Heilbut AM, Johansen LM, Price ER, et al. Synergistic drug combinations tend to improve therapeutically relevant selectivity. Nat Biotechnol. 2009;27(7):659-66.

14. Speranza MC, Nowicki MO, Behera P, Cho CF, Chiocca EA, Lawler SE. BKM-120 (Buparlisib): a Phosphatidyl-Inositol-3 kinase inhibitor with antiinvasive properties in Glioblastoma. Scientific reports. 2016;6:20189.

15. Rodon J, Brana I, Siu LL, De Jonge MJ, Homji N, Mills D, et al. Phase I doseescalation and -expansion study of buparlisib (BKM120), an oral pan-Class I PI3K inhibitor, in patients with advanced solid tumors. Invest New Drugs. 2014;32(4):670-81.

16. Ando Y, Inada-Inoue M, Mitsuma A, Yoshino T, Ohtsu A, Suenaga N, et al. Phase I dose-escalation study of buparlisib (BKM120), an oral pan-class I PI3K inhibitor, in Japanese patients with advanced solid tumors. Cancer Sci. 2014;105(3):347-53.

17. Maira SM, Pecchi S, Huang A, Burger M, Knapp M, Sterker D, et al. Identification and characterization of NVP-BKM120, an orally available pan-class I PI3-kinase inhibitor. Mol Cancer Ther. 2012;11(2):317-28.

18. Bijnsdorp IV, Giovannetti E, Peters GJ. Analysis of drug interactions. Methods Mol Biol. 2011;731:421-34.

19. Juric $D$, Castel P, Griffith M, Griffith OL, Won HH, Ellis H, et al. Convergent loss of PTEN leads to clinical resistance to a PI(3)Kalpha inhibitor. Nature. 2015;518(7538):240-4.

20. Tan X, Hu L, Luquette LJ 3rd, Gao G, Liu Y, Qu H, et al. Systematic identification of synergistic drug pairs targeting HIV. Nat Biotechnol. 2012;30(11):1125-30.

21. Mathews Griner LA, Guha R, Shinn P, Young RM, Keller JM, Liu D, et al. High-throughput combinatorial screening identifies drugs that cooperate with ibrutinib to kill activated B-cell-like diffuse large B-cell lymphoma cells. Proc Natl Acad Sci USA. 2014;111(6):2349-54.

22. Di Veroli GY, Fornari C, Wang D, Mollard S, Bramhall JL, Richards FM, et al. Combenefit: an interactive platform for the analysis and visualization of drug combinations. Bioinformatics. 2016;32(18):2866-8.

23. Jansen VM, Bhola NE, Bauer JA, Formisano L, Lee KM, Hutchinson KE, et al. Kinome-wide RNA interference screen reveals a role for PDK1 in acquired resistance to CDK4/6 inhibition in ER-positive breast cancer. Cancer Res. 2017;77(9):2488-99.

24. Pareja F, Macleod D, Shu C, Crary JF, Canoll PD, Ross AH, et al. PI3K and $\mathrm{BCl}-2$ inhibition primes glioblastoma cells to apoptosis through downregulation of Mcl-1 and Phospho-BAD. Mol Cancer Res. 2014;12(7):987-1001.

25. Lineham E, Tizzard GJ, Coles SJ, Spencer J, Morley SJ. Synergistic effects of inhibiting the MNK-elF4E and PI3K/AKT/mTOR pathways on cell migration in MDA-MB-231 cells. Oncotarget. 2018;9(18):14148-59.

26. Chen IC, Hsiao LP, Huang IW, Yu HC, Yeh LC, Lin CH, et al. Phosphatidylinositol-3 kinase inhibitors, buparlisib and alpelisib, sensitize estrogen receptor-positive breast cancer cells to tamoxifen. Scientific reports. 2017;7(1):9842.

27. Gad H, Koolmeister T, Jemth AS, Eshtad S, Jacques SA, Strom CE, et al. MTH1 inhibition eradicates cancer by preventing sanitation of the dNTP pool. Nature. 2014;508(7495):215-21.

28. Juvekar A, Hu H, Yadegarynia S, Lyssiotis CA, Ullas S, Lien EC, et al. Phosphoinositide 3-kinase inhibitors induce DNA damage through nucleoside depletion. Proc Natl Acad Sci USA. 2016;113(30):E4338-47.

29. Horvathova E, Dusinska M, Shaposhnikov S, Collins AR. DNA damage and repair measured in different genomic regions using the comet assay with fluorescent in situ hybridization. Mutagenesis. 2004;19(4):269-76.

30. Srivastava N, Gochhait S, de Boer P, Bamezai RN. Role of H2AX in DNA damage response and human cancers. Mutat Res. 2009;681(2-3):180-8.

31. Huang TT, Lampert EJ, Coots C, Lee JM. Targeting the PI3K pathway and DNA damage response as a therapeutic strategy in ovarian cancer. Cancer Treat Rev. 2020;86:102021. 
32. Aristizabal Prada ET, Orth M, Nolting S, Spottl G, Maurer J, Auernhammer C. The MTH1 inhibitor TH588 demonstrates anti-tumoral effects alone and in combination with everolimus, 5-FU and gamma-irradiation in neuroendocrine tumor cells. PLoS ONE. 2017;12(5):e0178375

33. Gul N, Karlsson J, Tangemo C, Linsefors S, Tuyizere S, Perkins R, et al. The MTH1 inhibitor TH588 is a microtubule-modulating agent that eliminates cancer cells by activating the mitotic surveillance pathway. Scientific Rep. 2019;9(1):14667

34. Gallia GL, Rand V, Siu IM, Eberhart CG, James CD, Marie SK, et al. PIK3CA gene mutations in pediatric and adult glioblastoma multiforme. Mol Cancer Res. 2006;4(10):709-14.

35. Oka S, Ohno M, Tsuchimoto D, Sakumi K, Furuichi M, Nakabeppu Y. Two distinct pathways of cell death triggered by oxidative damage to nuclear and mitochondrial DNAs. EMBO J. 2008;27(2):421-32.

36. Fujikawa K, Kamiya H, Yakushiji H, Fujii Y, Nakabeppu Y, Kasai H. The oxidized forms of dATP are substrates for the human MutT homologue, the hMTH1 protein. J Biol Chem. 1999;274(26):18201-5.

37. Pudelko L, Rouhi P, Sanjiv K, Gad H, Kalderen C, Hoglund A, et al. Glioblastoma and glioblastoma stem cells are dependent on functional MTH1. Oncotarget. 2017:8(49):84671-84.

38. Tu Y, Wang Z, Wang X, Yang H, Zhang P, Johnson M, et al. Birth of MTH1 as a therapeutic target for glioblastoma: MTH1 is indispensable for gliomatumorigenesis. Am J Translat Res. 2016;8(6):2803-11.
39. Ellermann M, Eheim A, Rahm F Viklund J, Guenther J, Andersson $M$, et al. Novel class of potent and cellularly active inhibitors devalidates MTH1 as broad-spectrum cancer target. ACS Chem Biol. 2017;12(8):1986-92.

40. Huber KV, Salah E, Radic B, Gridling M, Elkins JM, Stukalov A, et al. Stereospecific targeting of MTH1 by (S)-crizotinib as an anticancer strategy. Nature. 2014;508(7495):222-7

41. Koul D, Fu J, Shen R, LaFortune TA, Wang S, Tiao N, et al. Antitumor activity of NVP-BKM120-a selective pan class I PI3 kinase inhibitor showed differential forms of cell death based on p53 status of glioma cells. Clin Cancer Res. 2012;18(1):184-95.

42. Ibrahim YH, Garcia-Garcia C, Serra V, He L, Torres-Lockhart K, Prat A, et al. PI3K inhibition impairs BRCA1/2 expression and sensitizes BRCAproficient triple-negative breast cancer to PARP inhibition. Cancer Discov. 2012;2(11):1036-47.

\section{Publisher's Note}

Springer Nature remains neutral with regard to jurisdictional claims in published maps and institutional affiliations.
Ready to submit your research? Choose BMC and benefit from:

- fast, convenient online submission

- thorough peer review by experienced researchers in your field

- rapid publication on acceptance

- support for research data, including large and complex data types

- gold Open Access which fosters wider collaboration and increased citations

- maximum visibility for your research: over $100 \mathrm{M}$ website views per year

At BMC, research is always in progress.

Learn more biomedcentral.com/submissions 